\title{
EDITORIAL
}

\section{Diagnostic ultrasound widespread}

\author{
Hideyuki Hasegawa ${ }^{1}$
}

(C) The Japan Society of Ultrasonics in Medicine 2017

Owing to recent developments in hardware related to diagnostic ultrasound, the number of transducer elements in an ultrasonic probe has reached almost 10,000, and the scale of the acquisition hardware has also become significantly large. The result of this revolution is high-quality real-time volumetric imaging. This is one of the important directions for development of diagnostic ultrasound.

On the other hand, various kinds of portable ultrasound systems are being developed, which makes diagnostic ultrasound beneficial for people in a wider range of situations, not only medical use but also healthcare in general. Although the prevalence of diagnostic ultrasound is good news for those in the world of medical ultrasound, it might make diagnostic ultrasound seem like nothing special. Therefore, fundamental studies that significantly enhance the value of diagnostic ultrasound are now indeed demanded.

Various attempts have already begun in an effort to explore new possibilities of diagnostic ultrasound. One is the combination of ultrasound with other physical phenomenon, such as light. Photoacoustic imaging has been studied extensively in recent years, and it has now realized, for example, imaging of the network of small vessels at an extremely high spatial resolution, which cannot be done by only ultrasound. Diagnostic ultrasound imaging has also been fused with other modalities for medical imaging, such as computed tomography (CT) and magnetic resonance imaging (MRI).
Fusion of multiple modalities can aggregate the merits of the respective modalities. For example, the real-time capability of diagnostic ultrasound is suitable for surgery navigation, while it is difficult to use CT or MRI during surgery. However, lesion detectability of diagnostic ultrasound during surgery is improved by introducing the information from CT or MRI obtained in advance.

As described above, combining ultrasound with other physical phenomena or imaging modalities could be one of the directions for expanding the possibilities of diagnostic ultrasound. On the other hand, fundamental research that enhances and expands the capabilities of diagnostic ultrasound itself will also be important. I keenly hope that our society will be a place for various collaborations that will yield important developments in medical ultrasound. Fortunately, our society is composed of all kinds of peoplemedical doctors, engineers in academia and the corporate world, and other healthcare professionals, who have a hand in medical ultrasound in one way or another-and there should be various opportunities for collaborations. Also, the ultrasound-related hardware for various experimental investigations is now more easily available than before. The time has come. We hope that important new findings will come from our society and welcome you to report them in our journal.
Hideyuki Hasegawa

hasegawa@eng.u-toyama.ac.jp

1 Graduate School of Science and Engineering, University of Toyama, Toyama, Japan 